\title{
BEYOND THE UNIVERSITY: SIMULATION EDUCATION ON THE JOB
}

\author{
Peter Tag \\ Horizon Systems Modeling, LLC \\ 8201 Golf Course Road NW, Suite D-3 287 \\ Albuquerque, NM 87120, U.S.A.
}

\section{David Krahl}

Imagine That, Inc.

6830 Via Del Oro, Suite 230

San Jose CA 95119, U.S.A.

\begin{abstract}
Simulation modelers have a diversity of educational backgrounds including several engineering and scientific disciplines, mathematics and computer related fields. Many of the skills required to achieve modeling proficiency are learned "on the job". Emerging trends in the demand for more complex and fully automated simulation applications are requiring simulators to develop a working knowledge of a much broader range of software technologies and modeling methodologies. Unfortunately, there are no structured educational programs for acquiring and developing these skills. Simulation apprenticeships provide an effective means for acquiring many of the essential and emerging simulation skills that are not delivered through conventional educational methods.
\end{abstract}

\section{INTRODUCTION}

Simulation modeling is a discipline that is a mixture of art and science. It requires a variety of basic and advanced technical and non-technical skills. While most of the technical skills can be acquired by studying materials and taking formal training in fields such as industrial engineering, operations research, mathematics and computer science; non-technical skills must be acquired by other means. The path for acquiring these skills is quite circuitous and has several branches. As a simulator moves through different levels of modeling competency, different skills are required and/or accentuated. For a given model, the skills a modeler needs depend on several factors, e.g., the complexity of the system being modeled, the model users, the requirement to develop interfaces between the model and external systems, etc... Regardless of these factors, most models require simulators to have a common set of basic technical and non-technical skills. Naturally, as model complexity increases, the need for more advanced skills increases. However, there is an emerging trend for simulators to possess a much broader set of basic and advanced skills. This trend is the result of continuous advances in software and hardware technologies. These advances have increased the demand for larger and more complex simulation models and have engendered a new set of required simulation skills. As this trend continues, the means by which a simulator acquires these skills will become increasingly important.

\section{BACKGROUND}

Most modelers do not have a traditional industrial engineering or operations research background. A recent informal survey of active modelers on a popular simulation user's group (Extend E-Xchange, 2007) indicated that only $33 \%$ of active modelers have an academic background in industrial engineering or operations research.

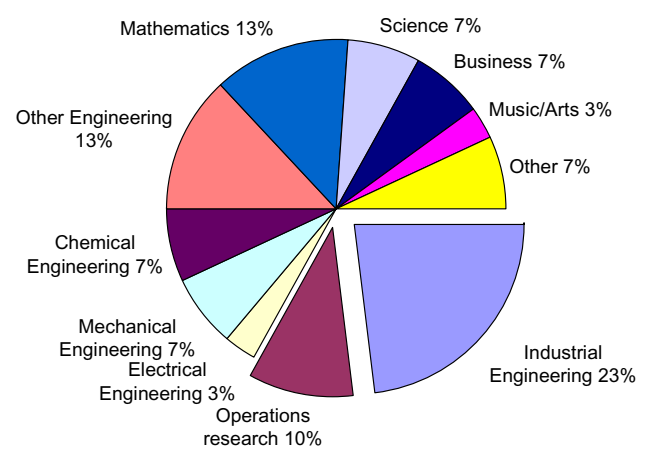

Figure 1: Academic Background of Simulation Modelers

Figure 1 shows the variety of education for simulation modelers. This chart indicates that most simulation analysts are trained "on the job" without the benefit of exposure to simulation modeling in college.

A good portion of the remainder has a background in mathematics, engineering, or computer science. While graduates from these programs have not had a course in discrete event simulation, they have probably been exposed to statistics and computer programming. 
For those who are industrial engineers, anecdotal information appears to indicate a trend away from programming requirements in industrial engineering programs. At two major industrial engineering schools, students are required to take one semester of programming and generally, do not reinforce this until their first simulation course two years later. In addition, the programming language that is required is not compatible with the simulation software that is used! In some cases, computer programming is not listed as a prerequisite for the required simulation course.

\section{SKILLS}

Much has been written in the last 10 years about the skills required for simulation modeling. Banks, 2001 provides a good set. However, the skills required are a bit like the parable of the blind men and the elephant (Wikipedia 2007). The nature of the model and the type of project will dictate the proficiencies required. A simulation consultant that builds many different kinds of models will need a broader range of skills than a plant floor industrial engineer that builds an occasional model to analyze a manufacturing process.

\subsection{Basic Skills}

Often a modeler is not a single person, but a team of modelers. Each person in this team brings a set of skills to the modeling process that compliments the others. One person may be a programmer that builds or modifies modeling components, a second team member assembles these components into a model, yet another works with the client setting the expectations and keeping the project on track.

Given the diverse community, what are the basic skills? At a minimum, there are three areas of proficiency to build simulation models:

- A basic understanding of the areas of statistics appropriate to simulation modeling. Specifically random distributions, confidence intervals, and design of experiments are required areas of proficiency.

- Some background in computer programming. Not because writing code is necessary for building models, but because as the modeler assembles a model, it is necessary to understand the basic mechanisms of the simulation program. Simulation models are collections and manipulations of data structures and algorithms. A modeler with knowledge of these computer science topics will build more reliable and higher quality models.

- Perhaps the most important skill is being savvy about the model and the modeling process. A savvy modeler will look for flaws in the model, navigate the politics of the project team, generally build understandable, straightforward models, and even recognize his or her own limitations.

\subsection{Advanced}

Continuous advances in computing technology open up new frontiers for simulation modelers. Historically, computer processing speeds increase at a rate predicted by Moore's Law. As processing speeds increase, the demands for more complex and larger scale models increase. Largescale simulation models engender a number of modeling challenges and issues that require advanced skills to effectively handle. For example, large-scale models require the management of much larger data sets than small-scale to medium-scale models. These models quickly exceed the threshold where input and output data can be manually managed in a timely and effective manner. Consequently, simulators must be capable of creating large-scale models that:

- Directly interface with data management systems

- Automate the population of model data structures from external data management systems

- Automate the delivery of model outputs to external data management systems

In order to design and create user-friendly data interfaces and automated data population mechanisms, simulators must have a basic understanding of database systems and client/server programming methodologies. Additionally, large-scale simulation models require well organized internal data-structures to facilitate auto-population mechanisms, process information efficiently and reduce model run-time. Often, the design of model data structures and relations in large-scale models can be more challenging than the logic representing model state transitions, i.e., system behavior.

Another challenge presented by large-scale simulation models is the representation of model logic to the model customer. Continuous advances in the graphical user interfaces of simulation tools provide simulators with opportunities to expose model customers to increasing amounts of model logic. The importance of this cannot be overstated. The more model logic that can be exposed to the model customer, the more trust the customer will have in the outputs from the model and the less time it will take to verify the model.

Another consequence of increased computing processing power is the demand for use of simulation models by non-modelers. Because simulation models can readily be connected to and auto-populated with data from external database systems, there is increasing demand for simulation models to become directly incorporated into regularly occurring decision-making processes where the simulator is out of the loop and simulation models are being run by non-simulators. For these situations, simulators need to be able to assemble integrated applications that allow non- 
simulators to cohesively perform data input, model execution and data output activities in a single interactive session.

\section{EDUCATIONAL RESOURCES}

If you do not have an Industrial Engineering or Operations Research background, it's a good idea to take a simulation class, if one is available, at a local university. From a course such as this, you are likely to learn the statistics and the basics of a simulation program. Vendor courses will teach you the details of a particular simulation tool. Many vendors are including some statistical analysis and an overview of the simulation process into even the basic classes. In addition, the last day of the course (or an additional day) is often reserved for answering questions on specific simulation models that are of interest to the students. Professional courses such as Averill Law's "Simulation Modeling for Systems Design and Analysis" can provide good coverage of the steps of a simulation study and statistics that are appropriate for simulation modeling.

Conferences, such as the Winter Simulation Conference or the Institute of Industrial Engineers Annual Conference are a good place to network with other simulation modelers, learn about simulation software, and attend a wide range of sessions from introductory tutorials to advanced simulation statistics and concepts.

If you are a book learner, there are a number of good books available. These include "Simulation Modeling and Analysis" (Law, 2007) and "Simulation Made Easy: A Managers Guide" (Harrell, 1995). Some simulation software manuals are excellent and are often available by downloading a demonstration version of the software. For the more advanced simulation modeler, "Theory of Modeling and Simulation" (Zeigler, 1976) is an excellent book. There are others. Find one that speaks to you.

Online resources such as simulation software forums (http://groups.google.com/group/comp.sim ulation/topics, www.extend-user.com, or www.simul8.com/cafe) can provide an insight into the modeling process. If the forum is sponsored by a software vendor, you may or may not need to be a customer to access the forum. Even if you are using a different software program, it can be useful to peruse forums oriented to other simulation products.

Another less obvious factor in successful modeling is the appropriate choice in software. Professional, full time modelers will be able to efficiently use simulation programs that routinely require programming. More occasional modelers will probably want to limit their choices to drag-and-drop software. Be careful not to equate cost with capability.

\section{THE SIMULATION APPRENTICE}

Apprenticeships in various forms exist for a wide variety of occupations. Of course many trades such as electricians and carpenters must go through an apprentice program before they can work independently. Medical doctors are interns before they practice on their own. Architectural apprentices are commonplace. Some engineering schools require a certain number of cooperative education internships with industry as a requirement for graduation.

Although there are different opinions on exactly what skills are required to be a successful modeler, no one will argue that a combination of skills is required. As an apprentice, a new modeler can work through projects under the watchful eye of someone more experienced.

\subsection{Apprenticeship Benefits}

There are a number of advantages of apprenticeships. Documented benefits (Employers for Apprentices, 2007) include:

- Positive return on investment

- Increased competitiveness

- Increased productivity

- Higher quality of work

- Reduced costs

- Improved recruitment

- Increased staff retention

- Higher employee satisfaction

- More potential for career progression

- More efficient use of new technology

- Supplementing ageing work force

- Widening the talent pool and increasing your appeal in diverse markets

Many of these, especially increased productivity, higher quality of work, and more efficient use of new technology apply directly to simulation modeling. Specifically an apprentice can learn techniques in model abstraction, determining the appropriate level of detail, validation, verification, and even the politics of the simulation project from someone with more modeling experience. These are not subjects that lend themselves to the academic environment. While a professor may certainly talk about these in a course, there is no substitute for experiencing them firsthand.

Cognitive apprenticeships (Collins, Brown \& Newman, 1987) allow a master simulator to model a decisionmaking process in a real-world context. As a master simulator models advanced simulation skills, the apprentice can quickly identify the knowledge and behaviors that are relevant for the development of effective models. This enables the apprentice to develop a conceptual model of the modeling processes.

Additionally, apprenticeships provide an efficient way for simulators to learn how to use and interface with many 
of the software technologies required for large-scale simulation models, e.g., relational databases, COM interfaces, GUI development environments, etc... Due to time constraints, it is impractical and less effective for simulators to learn all of these technologies by taking courses and reading books. Through apprenticeships, simulators can be exposed to the particular aspects of these technologies that are relevant to simulation modeling.

\subsection{Implementation of an Apprenticeship}

In a consulting organization, it is a standard procedure to pair less experienced employees with an experienced analysts. If you are getting started at your organization and do not have access to an internal simulation expert, we strongly recommend that you budget for consulting assistance as part of the cost of the simulation model. A good consultant can help you build the model and educate you on the simulation process. If you are in this situation, it is likely that your simulation models will be of a similar type and scope. Because of this, learning the modeling process once should easily translate into future models. Where beginning modelers have followed this process, they have generally become successful at the simulation process.

Projects requiring large-scale simulation models provide perfect opportunities for simulation apprenticeships. These projects have more budget and longer development schedules than small projects requiring small-scale to medium-scale simulation models.

If your organization is new to simulation, some simulation software companies as well as independent simulation modeling consulting organizations offer a mentoring service.

\subsection{Success Story}

A major aerospace company has implemented a program where new modelers work with a mentor on progressively more difficult modeling problems. These problems begin with textbook cases and progress into real-world applications. This has been successful in reducing the time required to train an analyst, improving the quality of the modeler's work, and identifying those who are not well suited to simulation modeling early in the process.

\subsection{Exceptions}

Of course, there are individuals who have a natural aptitude for simulation modeling and "see" the model without needing significant instruction or mentoring. Good examples are Martin Zand and his group at the University of Rochester (Zand, 2004) and Benjamin Wang and Robert Manning (Wang 2004) who have applied discrete event simulation in a new area of application with a minimum of input from experienced modelers. However, it is safe to comment that most new modelers would significantly benefit from working with an experienced simulationist.

\section{CONCLUSIONS}

A simulation modeler who is able to successfully model complex, real-world systems requires a range of skills not readily acquired by academic studies alone. Regardless of the background of the simulation modeler, an either formal or informal apprenticeship is recommended for getting started in simulation modeling.

\section{REFERENCES}

Banks, J., Panel Session: Education for Simulation Practice - Five Perspectives. In Proceedings of the 2001 Winter Simulation Conference, ed. B. A. Peters, J. S. Smith, D. J. Medeiros, and M. W. Rohrer, 1571-1579. IEEE, Piscataway, NJ

Barryman, S. E., Designing Effective Learning Environments: Cognitive Apprenticeship Models. IEE Brief, September 1991.

Collins, A., Brown, J. S., \& Newman, S. E. (1987). Cognitive apprenticeship: Teaching the craft of reading, writing and mathematics (Technical Report No. 403). BBN Laboratories, Cambridge, MA. Centre for the Study of Reading, University of Illinois. January, 1987.

Employers for Apprentices, Task Force Business Progress Report ATF/04/16 Part D Business Cases. <http: / / www . employersforapprentices. gov.uk/docs/meetings/Meeting_10_180. doc $>$. [Accessed July 10, 2007].

Extend E-Xchange 2007. Poll: What is your academic background.

<http://www. extenduser. com>. [Accessed July 10, 2007].

Harrell, C., and C. Tumay. 1995. Simulation made easy - a manager's guide. Norcross, Georgia, USA: Industrial Engineering and Management press.

Law, A. M., and W. D. Kelton. 2007. Simulation modeling \& analysis. 4th Ed. New York: McGraw-Hill, Inc.

Wang, B., \& Manning R. 1999. Computer Simulation Modeling for Recreation Management: A Study on Carriage Road Use in Acadia National Park, Maine, USA, in Environmental Management, Vol. 23, No., 2, 193-203.

Wikipedia: (2004) The Moore's Law. Available online via <http://en.wikipedia.org/wiki/Moore' s_law> [Accessed July 14, 2007].

Wikipedia: (2004) The Blindmen and the Elephant. Available online via <http://en.wikisource.org/wiki/The_B lindmen_and_the_Elephant> [Accessed June 29, 2007]. 
Zand M. S., Briggs BJ, Bose A, and Vo T. 2004. Discrete event modeling of $C D 4+$ memory $T$ cell generation. $\mathrm{J}$ Immunol 173(6):3763-3772

Zeigler, B. P. 1976. Theory of Modeling and Simulation New York: John Wiley and Sons.

\section{AUTHOR BIOGRAPHIES}

PETER TAG is the owner of Horizon Systems Modeling, LLC. He received a B.S. in Geological Sciences from Lehigh University and an M.S. in Geophysics from Cornell University. Peter develops simulation models, scheduling algorithms, data management tools and automated data transfer interfaces between relational databases and Extend simulation models. Additionally, Peter has extensive software application development experience having implemented a number of different applications in Visual Basic, $\mathrm{C} / \mathrm{C}++$, FORTRAN and Pascal. He has consulted with several businesses and government organizations including Procter \& Gamble, Citicorp, Pratt \& Whitney, Intel and Los Alamos National Laboratory. His email address is <phtag@horizonsystemsmodeling.com> and his web-site

<http://www.horizonsystemsmodeling.com/

$>$.

DAVID KRAHL is Vice President of Technical Sales with Imagine That, Inc. He received a MS in Project and Systems Management in 1996 from Golden Gate University and a BS in Industrial Engineering from the Rochester Institute of Technology in 1986. Mr. Krahl has worked extensively with a range of simulation programs including Extend, SLAM II, TESS, Factor, AIM, GPSS, SIMAN, XCELL+ and MAP/1. A few of the companies that Mr. Krahl has worked with as a consultant and educator are Chrysler, Ford, Williams International, Tefen, Raytheon, and Boeing. He is actively involved in the simulation community. His email address is <davek@extendsim.com> and the Imagine That Inc. site is <www. extendsim.com>. 